\title{
Key Factors Promoting Rapid Implementation of Virtual Screening Modalities for the COVID-19 Pandemic Response
}

Beth A. Careyva, MD, Grant Greenberg, MD, MHSA, MA, Robert Kruklitis, MD, PhD, Kyle Shaak, MPH, John J. Stoeckle, MD, CHQS, and Jennifer Stephens, DO, FACP

Background: The COVID-19 (C-19) pandemic required swift response from health care organizations to mitigate spread and impact. A large integrated health network rapidly deployed and operationalized multiple access channels to the community, allowing assessment and triage to occur virtually. These channels were characterized by swift implementation of virtual models, including asynchronous e-visits and video visits for $\mathrm{C}-19$ screening.

Purpose: (1) Evaluate implementation characteristics of C-19 screening e-visits and video visits. (2) Identify volume of $\mathrm{C}-19$ screening and other care provided via e-visits and video visits. (3) Discuss future implications of expanded virtual access models.

Methods: Retrospective analysis of implementation data for C-19 screening e-visits and video visits, including operational characteristics and visit/screening volumes conducted.

Results: Virtual channels were implemented and rapidly expanded during the first week C-19 testing was made available. During the study period, primary care clinicians conducted 10,673 e-visits and 31,226 video visits with 9,126 and 26,009 patients, respectively. Within these 2 virtual modalities, 4,267 C-19 tests were ordered (10\% of visits). Four hundred forty-eight clinicians supported 24/7 access to these virtual modalities.

Discussion: Given ongoing patient interest and opportunity, virtual health care services will continue to be available for an expanded number of symptoms and diagnoses. ( $\mathrm{J}$ Am Board Fam Med 2021;34:S55-S60.)

Keywords: CoVID-19, Health Technology, Pandemics, Primary Health Care, Retrospective Studies, Telemedicine

\section{Introduction}

The World Health Organization (WHO) recommends key actions occur before, during, and after a pandemic. Local actions include reducing the spread of disease and ensuring continuity of health care provision. Mitigating the spread of pandemic disease requires social distancing and infection control measures. ${ }^{1}$ The emergence of the COVID-19 (aka coronavirus) pandemic required more aggressive social

This article was externally peer reviewed.

Submitted 19 July 2020; accepted 5 August 2020.

From the Lehigh Valley Health Network, Department of Family Medicine, Allentown, PA (BAC, GG, JJS, KS); Lehigh Valley Health Network, Department of Medicine, Allentown, PÁ (JS, RK).

Funding: None.

Conflicts of interests: None.

Corresponding author: Beth A. Careyva, MD, Lehigh Valley Health Network, Department of Family Medicine, 3080 Hamilton Blvd Suite 250, Allentown, PA 18103 (Email: beth_a.careyva@lvhn.org). distancing given the highly communicable nature of the virus and subsequent lack of treatments, due to its novel characteristics. ${ }^{2}$ The need to keep people home while assessing symptoms and risk presented novel challenges, at the same time requiring a swift and innovative response from health care organizations. A large integrated health network rapidly deployed and operationalized multiple health care access channels to the local community, allowing patient assessment and triage to occur virtually. These channels were characterized by implementation of virtual models. including asynchronous e-visits and video visits for C-19 screening.

\section{Background \\ Virtual Channel Models}

Health care has increasingly become digital, with expansion of electronic health records (EHRs) 
within almost every hospital and office practice across the United States. In addition, patients are engaging more frequently with the health care system through online portals and communication channels. ${ }^{3}$ Models of virtual care are expanding but primarily include e-visits, video visits, and phone visits. A video visit includes both audio and visual communication during a single patient encounter. E-visits are characterized by an asynchronous message, like an e-mail, often leveraging templates for facilitation of clinical content and information transfer. General guidelines exist for virtual visits and include technology capabilities and security, patient consent, Health Insurance Portability and Accountability Act-compliant connectivity, documentation, and clinical care expectations. ${ }^{4}$

\section{Context}

Lehigh Valley Health Network (LVHN) is an integrated health network, serving a geographically diverse region in eastern Pennsylvania. Our organization provides care to approximately 400,000 people across rural, suburban, and urban environments in 5 counties.

The Lehigh Valley Physician Group is a nonprofit subsidiary medical group within LVHN composed of more than 1500 physicians and advanced practice clinicians. This group delivers comprehensive services in more than 200 practices, representing $60+$ medical specialties and completing more than 2.5 million visits/year.

The organization uses Epic, a vendor-based EHR, that is integrated across the entire continuum for documentation. Analytics employing advanced business intelligence tools are used, providing realtime performance metrics driven from EHR data. Virtual care has been functional at LVHN and scaled internally for many years to include scheduled ExpressCARE (urgent care) video visits and internal consultation services, including an advanced (remote) intensive care unit model. ${ }^{5}$ E-visits had been piloted in primary care previously but were not in use across LVHN at the time of C-19 onset.

\section{Methods}

The WHO declared C-19 a pandemic on March 11, 2020. The study period is defined as the time period between March 11, 2020 and May 31, 2020. Primary care is defined as ambulatory encounters conducted by family medicine, general internal medicine, and ExpressCARE clinicians. Both e-visits and video visits are considered virtual visits for the purpose of this study.

\section{Technology Infrastructure}

At the onset of C-19 within our community, the organization rapidly scaled our existing video platform and e-visit functionality in the EHR to provide community-facing virtual channels. The video visit model was augmented from a scheduled model supporting acute visits to one with a dedicated C-19 screening program with capacity for scheduled visits in addition to an on-demand queuing functionality. The e-visit functionality was transitioned to be focused on C-19 screening only, with encounters populating a centralized work queue. Templates for virtual encounters were built from network C-19 guidelines to standardize patient symptom evaluation and create a consistent clinical assessment. Laboratory systems, which use a different electronic medical record vendor than LVHN, developed an interface to accept C-19 test orders produced from virtual encounters so that patients would no longer require an article prescription. Patients were able to access the virtual channels through their smartphone by downloading the network's portal app. The Epic EHR portal prominently displayed C-19 video screening and e-visits through dedicated buttons to facilitate quick entry.

\section{Patient Engagement}

Community marketing was leveraged to inform access to these virtual channels. Our organization has historically engaged approximately 300,000 unique patients on the virtual portal. During the 6week study period, volume of new activations increased, with the peak occurring at 20,243 activations in April (Appendix) through this engagement and support. Multiple communication channels including modification of the LVHN website, social media messages, television and radio interviews, outdoor advertising, and print media were leveraged to describe virtual options for patients and to encourage the "Stay Home, Save Lives" message. The screening visits were also provided as a community benefit free of charge.

\section{Staffing Infrastructure}

C-19 screening e-visits were initiated within the first week of the pandemic by soliciting volunteers 
from our clinical workforce. This included both family medicine and internal medicine physicians and advanced practice clinicians who worked in outpatient settings in primary care and urgent care (ExpressCARE). Clinicians initially self-scheduled via an online scheduling program into shifts to ensure continuous coverage and timely response in less than 24 hours to the messages that flowed into a central work queue. Clinicians were able to select a message and "take the baton" within the EHR to communicate to other members of the team that the visit was in progress. Clinicians were able to conduct these visits off-site, given that no additional technology was required beyond access to the EHR. The number of volunteers was immediately robust, speaking to a culture of collaboration and commitment to the community in the face of an unprecedented crisis.

A similar staffing model was used for the C-19 screening video visits, with the number of clinicians per shift continuously monitored and adjusted to meet demand by leadership of the network's remote monitoring program. Initially, clinicians completed video visits out of an existing, centralized telehealth hub with scheduled visits, as per the prior model of acute care video visits. Within the first 3 weeks of the pandemic, workflows were developed to allow clinicians to complete these visits off-site, using a centralized pool and on-demand queue model within the EHR. This queuing model allowed increased flexibility with scheduling and ability for a larger number of clinicians to participate without having to sign up for a full "shift" of set hours, including individuals who had C-19 exposure and were under mandatory quarantine who otherwise would not have been able to care for patients in a face-to-face manner.

\section{Results}

\section{Encounter Volumes}

Before C-19, in 2020 (January 1 through March 10), primary care conducted 39 virtual visits. During the 11-week study period, 10,673 e-visits and 31,226 video visits were completed, for a total of 41,899 virtual visits. Of these visits, 12,746 were conducted for C-19 screening for patients expressing symptoms consistent with C-19. Video visits made up $60 \%$ ( $n=$ 7,688 ) of C-19 screening virtual visits, and $40 \%$ ( $\mathrm{n}=$ 5,058) were e-visits. C-19 tests for 4,267 were ordered as a result of these C-19 virtual visits, for an overall testing rate of $33 \%$ (Table 1 ).
A subset of all video visits (6430) exclusively designed for C-19 screening were completed using the on-demand work queue process. Of these visits, $83 \%(\mathrm{n}=5359)$ had a documented primary diagnosis of a C-19 related symptom. A C-19 test was ordered during $35 \%(n=2275)$ of these video visits. Two hundred twenty-two clinicians completed video visits through the on-demand modality. Visits on Saturdays and Sundays accounted for 21\% ( $\mathrm{n}=$ 1324) of on-demand video visits.

\section{Discussion}

Rapid expansion of C-19 screening e-visits and video visits was feasible within a large health network. Doing so provided safe screening and care to a community acutely devastated by illness, fear, and anxiety. As opposed to many current direct-to-consumer telemedicine services available, ${ }^{6}$ ours was staffed entirely with local clinicians, and we were actively able to monitor and address quality and ensure consistent and timely response to facilitate robust identification of C-19 within our community.

\section{Cballenges}

Given the need to implement these modalities quickly and to manage the volume, a large number of clinicians were needed to operationalize C-19 screening e-visits and video visits. As ambulatory primary care volumes decreased, a voluntary workforce was shifted to staff these visits 24/7. This care could have been provided by a smaller number of clinicians providing an increased number of hours, but the ability to take a few visits from the e-visit or video visit queue throughout the day allowed for decreased time to response and increased flexibility for clinicians, some of whom completed these in between scheduled visits with their own patients.

\section{Table 1. CoVID-19 Visit and Encounter Types}

\begin{tabular}{|c|c|c|c|}
\hline & E-Visits & $\begin{array}{l}\text { Video } \\
\text { Visits }\end{array}$ & $\begin{array}{c}\text { Total } \\
\text { Virtual } \\
\text { Visits }\end{array}$ \\
\hline $\begin{array}{l}\text { Encounters with COVID-19 } \\
\text { symptom as primary diagnosis }\end{array}$ & 5,058 & 7,688 & 12,746 \\
\hline $\begin{array}{l}\text { Encounters with COVID-19 test } \\
\text { ordered }\end{array}$ & 1,385 & 2,882 & 4,267 \\
\hline $\begin{array}{l}\text { Encounters on Saturday or Sunday } \\
\text { for COVID-19 screening }\end{array}$ & 2,029 & 1,551 & 3,580 \\
\hline Number of providers & 258 & 435 & 448 \\
\hline
\end{tabular}


Managing an appropriate number of staff proved challenging. Some time slots were overstaffed and other times were more difficult to fill, and the inconsistences with both staffing and visit volume resulted in variability in wait times. Overall, there was minimal need for overnight staffing. Constraints in our ability to provide testing to appropriate patients led to multiple iterations of protocol and guidance. The rate of test ordering most definitely varied as the protocol for testing, availability of testing, and access points for testing evolved during the course of the study period. Not all suspected COVID cases were sent for testing during certain periods, but some were diagnosed empirically due to limited testing capacity and counseled accordingly. Frequent communication, often limited to e-mail, was the primary venue to convey protocol changes to the workforce. For e-visits, development of a template with iterative changes was needed to standardize the approach and to effectively obtain needed information while limiting need for back-and-forth communication. Within the study period, this template underwent multiple improvements in response to feedback from the clinician workforce.

Patient challenges included the need for interpreter services and technology difficulties. Interpreter services were accessed either locally or through a telephonic service when a bilingual clinician was not available. For those patients with technology challenges, some visits initially scheduled as video visits were converted to phone visits. These visits were excluded from the total number of completed video visits.

\section{Opportunities/Future Areas of Focus}

This study demonstrated capacity to stand up a model of timely population health management for C-19 screening that could be deployed for future pandemics. These virtual care modalities enabled needed care while preserving physical distancing unless testing was indicated, which was completed at assess and test centers, separated from ambulatory practices. For those patients who required screening, a process was developed during the study to integrate orders, thus removing the barrier of a needed article lab slip. This decreased risk of further exposure and removed an additional barrier to completion of C-19 testing.

The ability to access virtual care may not be universal among the population we serve. Financial constraints, access to Internet or Internet-enabled devices, or barriers to communication (language, developmental) are likely to skew the benefit of virtual care to a more limited patient demographic. In particular, given the racial disparities reported in C-19 cases nationally, measuring, identifying, and closing any gaps in access with virtual modalities of care will be an essential future development. ${ }^{7}$

The swift creation of a centralized workforce model for both e-visits and video visits enabled guideline-concordant care and a standard process to determine need for C-19 screening. Furthermore, it provided a mechanism to facilitate socially distant access to care for the population that may otherwise have been reluctant or unable to seek care. The rapid uptake of virtual care in our community provides an opportunity to remove barriers to access in our rural and more vulnerable communities longer term. $^{8}$ Previous studies have demonstrated that patients appreciate the convenience, efficiency, communication, privacy, and comfort afforded by virtual care. ${ }^{9}$

Given the robust patient response to these visit types, e-visits and on-demand video visits continue to be deployed for acute care with an expanded number of symptoms/diagnoses. Although much of this care is already being transitioned back to the primary care clinicians to support continuity of care, the centralized care delivery model may have a future role within the medical group for specific visit types, such as after-hours coverage, transition of care visits, and/or preoperative risk assessment.

Use of virtual care was most popular during the weekend days, which also highlights an opportunity to continue to support access via virtual channels to deliver care 7 days per week.

A process to implement large-scale virtual care, in any other time, would have taken years. The rapid implementation required senior leadership engagement and approval, alignment of proposals with fiscal goals, development of clinical workflows, and training of both clinical staff and patients. ${ }^{10}$ Accelerated by the pandemic, the swift pivot in care delivery was unprecedented. This demonstrates that not all innovation requires slow speed, pilot testing, and tempered growth. However, the key moving forward will be to integrate virtual care into a new "business as usual" model. ${ }^{11}$

\section{Conclusion}

C-19 screening e-visits and video visits promoted timely and centralized access to care. These visits 
facilitated appropriate C-19 screening to those patients who required testing and further information and/or reassurance to those who did not meet testing criteria. These technologies initially required a large centralized workforce that was adjusted iteratively to meet demand.

To see this article online, please go to: bttp://jabfm.org/content/ 34/Supplement/S55.full.

\section{References}

1. World Health Organization. Pandemic influenza preparedness and response: a WHO guidance document. 5, Recommended actions before, during and after a pandemic. 2009. Available from: https:// www.ncbi.nlm.nih.gov/books/NBK143063/.

2. Cascella M, Rajnik M, Cuomo A, et al. Features, evaluation, and treatment of coronavirus (COVID19). May 18, 2020. Available from: https://www. ncbi.nlm.nih.gov/books/NBK554776/.

3. Trends in individuals' use of health IT. 2020. Available from: https://dashboard.healthit.gov/ quickstats/quickstats.php. Accessed June 15, 2020.

4. American Academy of Family Physicians. Virtual evisits. 2020. Available from: https://www.aafp.org/ about/policies/all/virtual-visits.html. Accessed June 15,2020 .
5. McCambridge $\mathrm{M}$, Jones $\mathrm{K}$, Paxton $\mathrm{H}$, et al. Association of health information technology and teleintensivist coverage with decreased mortality and ventilator use in critically ill patients. Arch Intern Med 2010;170:648-53.

6. Uscher-Pines L, Mulcahy A, Cowling D, et al. Access and quality of care in direct-to-consumer telemedicine. Telemed J E Health 2016;22: 282-7.

7. Centers for Disease Control and Prevention. COVID19 in racial and ethnic minority groups. 2020. Available from: https://www.cdc.gov/coronavirus/2019-ncov/ need-extra-precautions/racial-ethnic-minorities.html. Accessed July 17, 2020.

8. Marcin J, Shaikh U, Steinhorn R. Addressing health disparities in rural communities using telehealth. Pediatr Res 2016;79:169-76.

9. Powell R, Henstenburg J, Cooper G, et al. Patient perceptions of telehealth primary care video visits. Ann Fam Med 2017;15:225-9.

10. Ellimoottil C, An L, Moyer M, Sossong S, Hollander J. Challenges and opportunities faced by large health systems implementing telehealth. Health Aff (Millwood) 2018;37:1955-9.

11. Jury S, Kornberg A. Integrating telehealth into "business as usual": is it really possible? J Telemed Telecare 2016;22:499-503. 


\section{Appendix.}

Figure 1. Electronic health record portal activations (July 2019 - June 2020).

MyLVHN Activations

Patients are currently Active on MyLVHN. Data last updated 6/24/2020 6:43:57 AM.

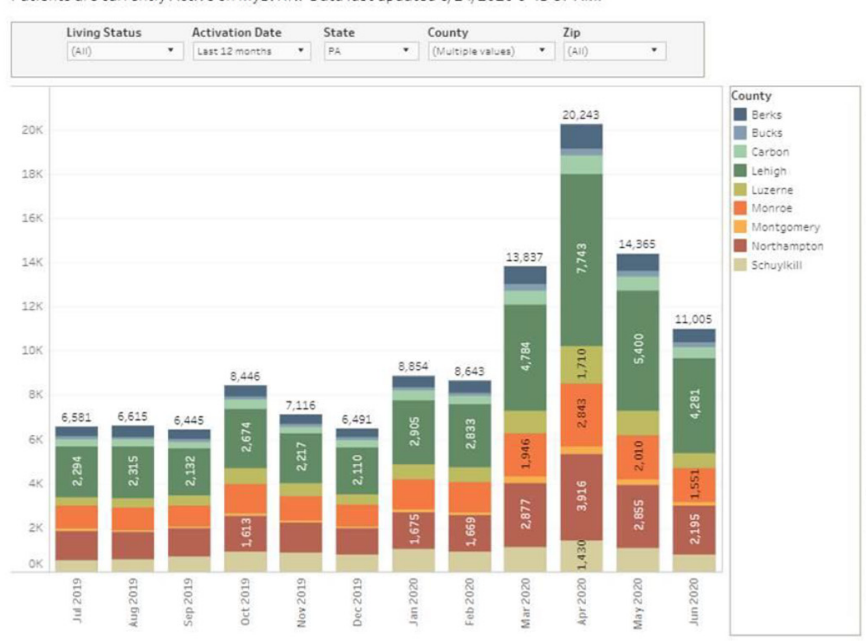

(2) Data by Enterprise Analytics

Total Current Activations $\quad 396,295$

Total Activations (Filtered) $\quad 118,641$ 